L

\title{
ANÁLISE NUMÉRICA DE INSTABILIDADE LATERAL EM VIGA PRÉ-MOLDADA ESBELTA
}

\author{
Buckling numerical analysis in slender beam with ANSYS
}

\author{
Maria Teresa Santos do Amaral Cardoso ${ }^{1}$, Maria Cristina Vidigal de Lima ${ }^{2}$
}

Recebido em 21 de fevereiro de 2017; recebido para revisão em 18 de abril de 2017; aceito em 25 de maio de

2017; disponível on-line em 18 de agosto de 2017.

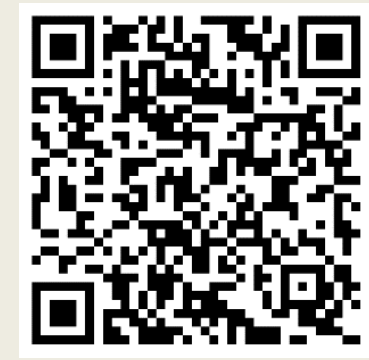

PALAVRAS CHAVE:

Vigas esbeltas;

Instabilidade;

Carga crítica;

Içamento;

Apoios deformáveis e

indeformáveis.

\section{KEYWORDS:}

Slender beams;

Instability;

Critical load;

Lifting;

Deformable or not

deformable supports.
RESUMO: A utilização de vigas pré-moldadas de concreto cada vez mais esbeltas tem se tornado comum na Engenharia Civil, sobretudo aquelas destinadas a transpor longos vãos em pontes, viadutos e obras de arte em geral. Preocupa o comportamento dessas vigas face à estabilidade lateral nas fases transitórias e em serviço. A presença de imperfeições geométricas agrava ainda mais a situação crítica dessas vigas. Duas fases devem ser avaliadas: o içamento e quando a viga encontra-se sobre apoios provisórios, ou seja, quando as ligações definitivas ainda não foram executadas. Na tentativa de estudar o comportamento de vigas esbeltas com relação à estabilidade lateral, este trabalho apresenta a análise de instabilidade feita numa viga de dimensões padronizadas denominada viga platibanda realizada no programa computacional de análise numérica ANSYS. O objetivo é conhecer a carga crítica de instabilidade para cada uma das situações analisadas: içamento com cabos retos e inclinados na presença e na ausência de balanços e quando a viga encontra-se apoiadas sobre apoios tipo garfo deformáveis ou não a torção na presença e na ausência de balanços. A determinação da carga crítica de flambagem permitiu conhecer os casos que conferem mais segurança à estrutura durante as fases transitórias. Dos casos analisados, a suspensão com cabos retos e balanços e a situação da viga sobre apoios indeformáveis a torção mostraram ser os casos mais benéficos para a segurança da estrutura.

ABSTRACT: Using prestressed slender beams has become common in Civil Engineering, especially those designed to transpose long spans of bridges and viaduct. It is worrying behavior of these beams due to the lateral stability in the transitional phases and in service. The presence of geometric imperfections aggravates the situation of these beams. Two stages must be evaluated: lifting and when the beam is on provisional supports, i.e., when final connections are not yet executed. In order to study the behavior of slender beams with respect to lateral stability, this work presents the eigenbuckling analysis made in a beam of standard dimensions called "viga platibanda" performed in the computational program of numerical analysis ANSYS. The objective is to determine the critical load of instability for each of the analyzed situations: lifting with straight and inclined cables in the presence and in the absence of overhangs and when the beam is supported by deformable or not deformable supports in the presence and in the absence of overhangs. The determination of the critical buckling load allowed to know the cases that give more security to the structure during the transitional phases. From all the analyzed situations, lifting with straight cables and overhangs and beam on indeformable supports proved to be the most beneficial cases for the safety of the structure.

\footnotetext{
* Contato com os autores:

${ }^{1}$ e-mail: mariateresasacardoso@gmail.com (M. T. S. A. Cardoso)

Mestre, Pós-Graduação em Engenharia Civil, Faculdade de Engenharia Civil, Universidade Federal de Uberlândia (UFU).

2e-mail: macris@ufu.br (M. C. V. Lima)

Doutora, Professora Associada 4, Faculdade de Engenharia Civil, Universidade Federal de Uberlândia (UFU).
} 


\section{INTRODUÇÃO}

Nos últimos anos, tem-se aumentado o comprimento de vigas de concreto a fim de atingir vãos maiores. Tendo em vista as limitações do transporte e dos equipamentos de içamento de peças pré-moldadas e a fim de reduzir o peso do elemento, a seção transversal dessas vigas tem se tornado cada vez mais esbelta, o que confere a elas uma baixa rigidez no eixo de menor inércia. Dessa forma, devido à presença de imperfeições iniciais, essas vigas são suscetíveis a flambagem lateral podendo ou não ser acompanhada de torção nas fases de içamento ou mesmo quando a viga encontra-se sobre apoios transitórios quando ainda nenhuma ligação foi efetivada.

A esbeltez é uma característica importante desses elementos lineares e que coloca em evidência um problema que tem se tornado recorrente no projeto e na construção das estruturas de grande porte: a instabilidade lateral durante as fases de içamento e de pré-serviço (considerada aqui, como a situação em que a viga está sobre os apoios, porém, com as ligações definitivas ainda não executadas). 0 comportamento mecânico de vigas delgadas é diferente do de vigas não delgadas. Vigas muito esbeltas e vigas moderadamente esbeltas estão sujeitas aos efeitos de instabilidade lateral próprios das dimensões de sua seção transversal (GIRIJA; MENON, 2011).

Deve-se mencionar também a influência das imperfeições geométricas na instabilidade lateral. Essas imperfeições podem ser decorrentes de erros ou falhas inevitáveis na concretagem, na aplicação da protensão e no posicionamento das alças de içamento. Esses desvios contribuem para a instabilidade lateral das vigas esbeltas, pois criam excentricidades adicionais que intensificam os efeitos decorrentes das fases transitórias.

$O$ estudo da estabilidade lateral de vigas metálicas é muito mais amplo que o de vigas de concreto. Há uma maior quantidade de verificações a serem realizadas em vigas metálicas. Embora o estudo das vigas metálicas possa servir de referência para as vigas de concreto, algumas diferenças são importantes de serem consideradas: o peso próprio das vigas de concreto é muito mais significativo, a rigidez à torção é muito superior quando comparada à rigidez em seu eixo de menor inércia e a aplicação da protensão não permite que a viga seja apoiada em grandes distâncias em relação às extremidades (STRATFORD; BURGOYNE, 1999).

\section{OBJETIVO}

O objetivo deste trabalho é apresentar uma análise de instabilidade (eigenbuckling) realizada no ANSYS em uma viga de dimensões padronizadas pela Associação Brasileira da Construção Industrializada ( $\mathrm{ABCl})$, denominada viga platibanda ou viga testeira. São analisadas, neste trabalho, diferentes situações de içamento e casos em que a viga está sobre apoios deformáveis e indeformáveis à torção. Para cada caso, determinase a carga crítica de instabilidade.

\section{REVISÃO BIBLIOGRÁFICA E CONTEXTUALIZAÇÃO}

O estudo da instabilidade lateral de vigas pré-moldadas de concreto é de extrema relevância e importância, haja vista os relatos de acidentes já ocorridos. Tremblay e Mitchell (2006) estudaram o tombamento de uma viga durante a construção de uma ponte de $144 \mathrm{~m}$ de comprimento em quatro vãos no Canadá. Oesterle et al. (2007) relataram o colapso de nove das 11 vigas que faziam parte de um dos 17 vãos de uma rodovia nos Estados Unidos devido à insuficiência do contraventamento em impedir o tombamento de uma das vigas e, consequentemente, o colapso progressivo das demais. Bairán e Cladera (2014) apresentaram a situação de colapso de uma viga de um sistema de cobertura, relacionado com a instabilidade lateral do elemento.

Os primeiros estudos que trataram com maior enfoque da estabilidade lateral de vigas durante o içamento foram realizados na década de 1960 por Swann e Godden (1966) e Massey e Walter (1969) e nas décadas de 1980 e 1990 por Imper e 
Lazlo (1987) e Mast (1989, 1993). Nos primeiros estudos, os autores se preocuparam em entender e explicar o problema e obter uma formulação mais prática que lhes permitisse aplicá-las em verificações mais simples, como a obtenção de fatores de segurança e a determinação das tensões em diferentes posições da viga. Experimentalmente, Massey e Walter (1969) simularam em escala reduzida vigas esbeltas sujeitas a flexão bi-lateral (Figura 1).

$\mathrm{O}$ assunto foi também tratado por Plaut e Moen (2013) e Cojocaru (2012). Plaut e Moen (2013) realizaram um estudo mais complexo ao considerar torção uniforme e torção não uniforme na viga ao ser suspensa. A formulação analítica proposta pelos autores deu origem a uma planilha de determinação dos esforços no içamento apresentada por Moen (2014) e utilizada por Cojocaru (2012).

A instabilidade lateral de vigas sobre apoios deformáveis foi estudada por Burgoyne e
Stratford (2001) e Plaut e Moen (2014). A partir de equações de equilíbrio, os trabalhos dos autores avaliaram analiticamente o problema e a influência da rigidez do apoio na estabilidade da estrutura.

Na literatura nacional, Lima (1995), Lima (2002) e Krahl (2014) preocuparam-se com a estabilidade lateral e investigaram o comportamento de elementos esbeltos prémoldados. Lima (1995) trabalhou com a formulação analítica para a carga crítica de instabilidade e estimou seus valores para a fase de içamento com cabos retos e inclinados na presença e na ausência de balanços e para a viga sobre apoios deformáveis e indeformáveis à torção. Lima (2002) analisou numérica e experimentalmente vigas sujeitas a tombamento lateral a fim de simular o comportamento dessas vigas sob flexão bi-lateral e torção. Krahl (2014) apresentou formulações para o cálculo da carga crítica de instabilidade lateral, do momento crítico e do fator de segurança.

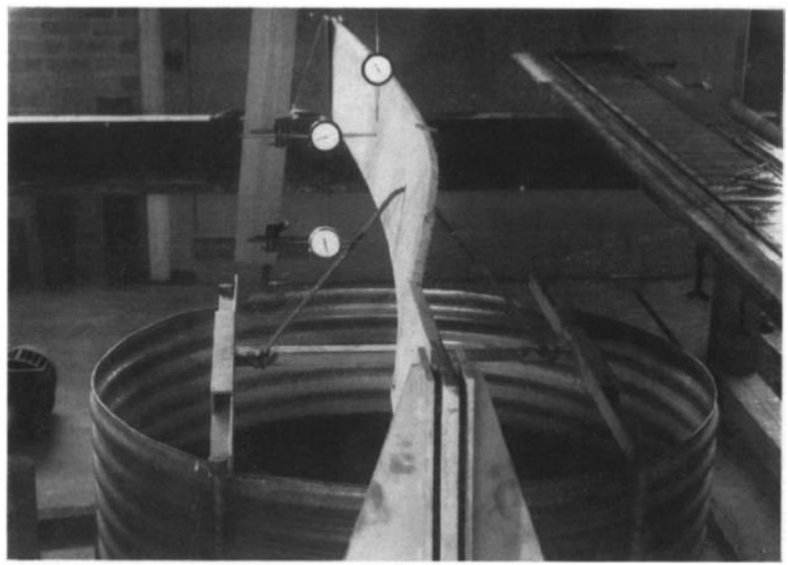

FIGURA 1: Ensaio em escala reduzida de viga esbelta sujeita a flexão bi-lateral.

FONTE: Massey e Walter (1969).

\section{METODOLOGIA}

Para a análise numérica realizada neste trabalho, utilizou-se o programa computacional de modelagem numérica ANSYS que tem como fundamento o Método dos Elementos Finitos. A análise de flambagem linear (ou eigenbuckling) permite conhecer a carga teórica de flambagem, ou seja, o ponto de bifurcação do equilíbrio, de uma análise elástica linear. Como resposta gerada pelo ANSYS nesta análise, tem-se um valor conhecido como FACT (load factor). Esse fator representa quantas vezes o carregamento da estrutura pode ser superado para se atingir uma configuração instável. Uma estrutura pode ter vários valores para FACT (load factors) associados a cada modo de flambagem (number of modes to extract) requerido, porém, o primeiro é usualmente o mais importante (ANSYS, 2017).

Para a análise deste trabalho, foram escolhidos três tipos de elementos: um elemento de viga (BEAM188), linear com seis graus de liberdade 
por nó (rotações e translações em $x, y$ e $z$ ); e dois elementos sólidos (SOLID65 e SOLID73). Ambos os elementos sólidos possuem oito nós com três graus de liberdade por nó (translações em $x, y$ e $z$ ), porém o elemento SOLID73 admite como grau de liberdade adicional a rotação na direção longitudinal $(x)$.

As condições de contorno adotadas são apresentadas em detalhes na Seção 5. Todas as análises de instabilidade (eigenbuckling) devem ser precedidas de uma análise estática linear. Um ponto importante em simulações numéricas é a escolha da malha adequada para o modelo. Nos casos apresentados neste trabalho, a malha adotada foi escolhida após testar malhas mais refinadas e obterse uma diferença considerada pequena pelas autoras.

\section{ANÁLISE DE INSTABILIDADE NA VIGA PLATIBANDA}

A viga platibanda possui seção transversal retangular 0,20 $\mathrm{m} \times 1,65 \mathrm{~m}$ (apresentada na
Figura 2) e $20 \mathrm{~m}$ de comprimento. As propriedades da viga platibanda são apresentadas na Tabela 1. Essa mesma viga foi analisada por Lima (1995).

O único carregamento ao qual a viga estava submetida era o peso próprio. A análise de instabilidade feita no ANSYS consistiu numa análise estática seguida de uma análise de flambagem (eigenbuckling). Para todos os casos analisados, definiram-se quatro modos de flambagem (oito para entrada no programa computacional).

Analisou-se, mais precisamente, o fator FACT, resultado do modelo numérico. Esse fator permite estimar a carga crítica de flambagem, uma vez que ele representa quantas vezes o carregamento considerado pode ser superado de modo a produzir um carregamento crítico que gera instabilidade na viga.

Foram modeladas duas situações distintas às quais a viga está sujeita no início de sua vida útil: o içamento e a fase de pré-serviço.

A Figura 3 apresenta a orientação dos eixos coordenados adotada nos modelos numéricos.

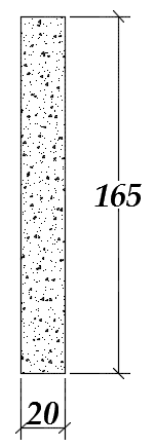

FIGURA 2: Seção transversal da viga platibanda (dimensões em cm).

FONTE: Autores (2017).

TABELA 1: Dimensões e propriedades mecânicas da viga platibanda.

\begin{tabular}{lc} 
Comprimento & $20 \mathrm{~m}$ \\
\hline Seção transversal & $0,20 \mathrm{~m} \times 1,65 \mathrm{~m}$ \\
\hline Módulo de elasticidade longitudinal do concreto & $3460 \mathrm{kN} / \mathrm{cm}^{2}$ \\
\hline Módulo de elasticidade transversal do concreto & $1440 \mathrm{kN} / \mathrm{cm}^{2}$ \\
\hline Peso próprio & $0,0825 \mathrm{kN} / \mathrm{cm}$ \\
\hline Momento de inércia à flexão lateral $\left(I_{y}\right)$ & $110000 \mathrm{~cm}^{4}$ \\
\hline Momento de inércia à flexão vertical $\left(I_{z}\right)$ & $7486875 \mathrm{~cm}^{4}$ \\
\hline Momento de inércia à torção $(J)$ & $440000 \mathrm{~cm}^{4}$ \\
\hline & FONTE: Autores (2017).
\end{tabular}




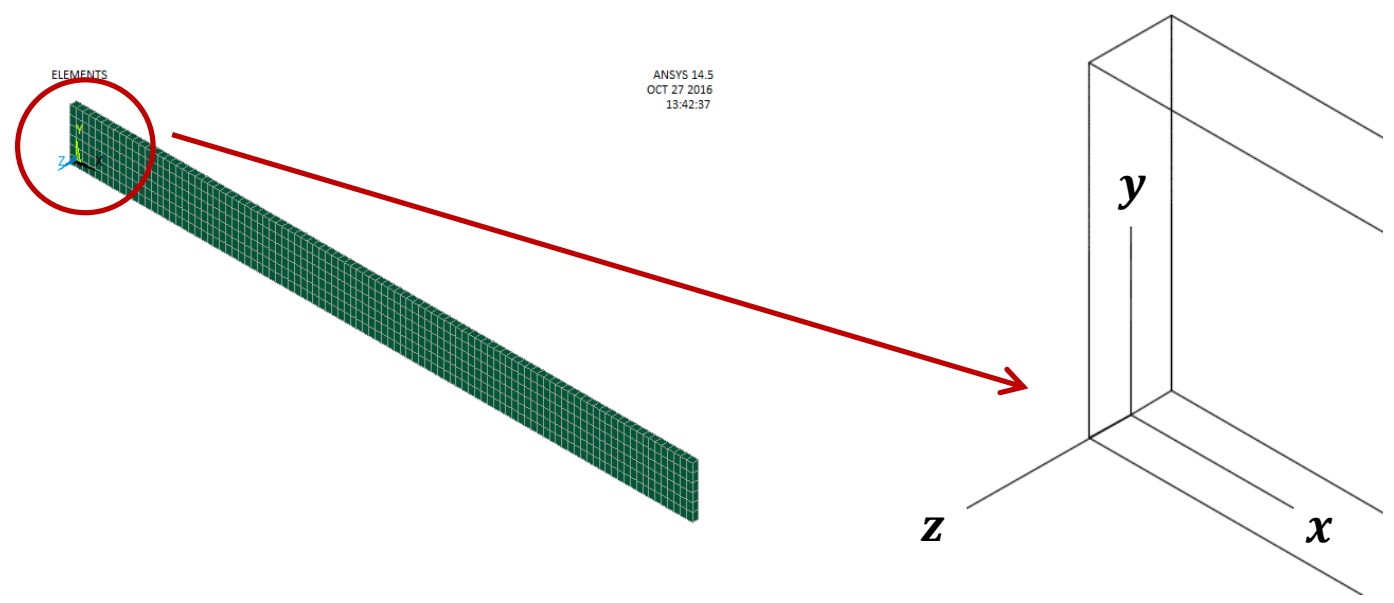

FIGURA 3: Orientação dos eixos coordenados.

FONTE: Autores (2017).

\subsection{IÇAMENTO}

No içamento, considerou-se que a viga seria suspensa sem balanços com cabos retos e com cabos inclinados, e com balanços e cabos retos. Os cabos rígidos não foram feitos nos modelos. Introduziram-se vinculações que fornecessem o mesmo efeito proporcionado pelos cabos. É importante destacar que os cabos não conferem à estrutura nenhum impedimento ao giro.

$\mathrm{Na}$ primeira análise, considerou-se o içamento com cabos retos sem balanços. As condições de contorno aplicadas aos modelos com elementos SOLID65 e SOLID73 foram as mesmas. Em uma das extremidades da viga, nos nós superiores, foram impedidos os deslocamentos UX, UY e UZ. Na outra, impediu-se UY e UZ. Para que a análise com elemento BEAM188 apresentasse resultados coerentes e que a viga não apresentasse um giro de corpo rígido, foi necessário impor em uma das extremidades, além das restrições aos deslocamentos UX, UY e UZ, a restrição ao giro ROTX. Na outra extremidade, manteve-se o impedimento aos deslocamentos UY e UZ.

$O$ içamento com cabos inclinados sem balanços foi a segunda análise. $O$ efeito da força de içamento no cabo foi tomado pela sua componente horizontal $(69,226 \mathrm{kN})$ que foi aplicada diretamente na viga. Essa componente horizontal corresponde ao ângulo de inclinação do cabo de $40^{\circ} \mathrm{com}$ a vertical. Para o modelo com elemento BEAM188, além da aplicação da componente horizontal, considerou-se também a atuação do momento fletor correspondente a essa componente em relação ao centroide da viga no valor de 5711,15 $\mathrm{kN} \cdot \mathrm{cm}$. Neste caso, em uma das extremidades da viga, foram impedidos os deslocamentos UX, UY e UZ e o giro ROTX. Na outra, impediu-se os deslocamentos UY e UZ. Com relação aos elementos sólidos, as condições de contorno impostas a cada um deles foi diferente. No modelo com elemento SOLID73, no nó superior intermediário de uma extremidade, foram impedidos os deslocamentos UX, UY e UZ e o giro ROTX. No nó correspondente da outra extremidade, foram impedidos os deslocamentos UY e UZ. Para o elemento SOLID65, o efeito do impedimento à rotação foi obtido restringindo-se o deslocamento UY dos nós superiores das duas extremidades. Mantiveram-se as demais condições de contorno do modelo com elemento SOLID73, ou seja, no nó superior intermediário de uma extremidade a restrição ao deslocamento UX e UZ e, na outra, a restrição ao deslocamento UZ.

O terceiro caso estudado foi a suspensão com cabos retos com balanços. Foi considerado que o cabo estava a uma distância equivalente aos quartos de vão das extremidades. Como na suspensão com cabos retos sem balanços, as condições de contorno aplicadas aos modelos com elementos SOLID65 e SOLID73 foram as mesmas. 0 deslocamento UY foi impedido em todos os nós superiores dos balanços. No nó central de um dos balanços, foi imposta a restrição aos deslocamentos UX e UZ. No outro balanço, também no nó central, 
restringiu-se apenas UZ. Semelhante ao que foi feito para a suspensão com cabos retos sem balanços modelada com elemento BEAM188, foi necessário impor em um dos balanços, além das restrições aos deslocamentos UX, UY e UZ, a restrição ao giro ROTX. No outro balanço, manteve-se o impedimento aos deslocamentos UY e UZ.

A Tabela 2 resume os valores de carga crítica obtidos para cada caso analisado na fase transitória de suspensão e de acordo com o tipo de elemento utilizado no modelo.

A Figura 4 apresenta as configurações deformadas dos quatro modos de flambagem para a viga platibanda em fase transitória de suspensão com cabos retos sem balanços modelada com elemento SOLID73 e as respectivas cargas críticas de instabilidade lateral.

Os valores de carga crítica apresentados na Tabela 2 mostraram que, dentre os casos de içamento analisados, a suspensão sem balanços e com cabos inclinados é a mais crítica para a viga,

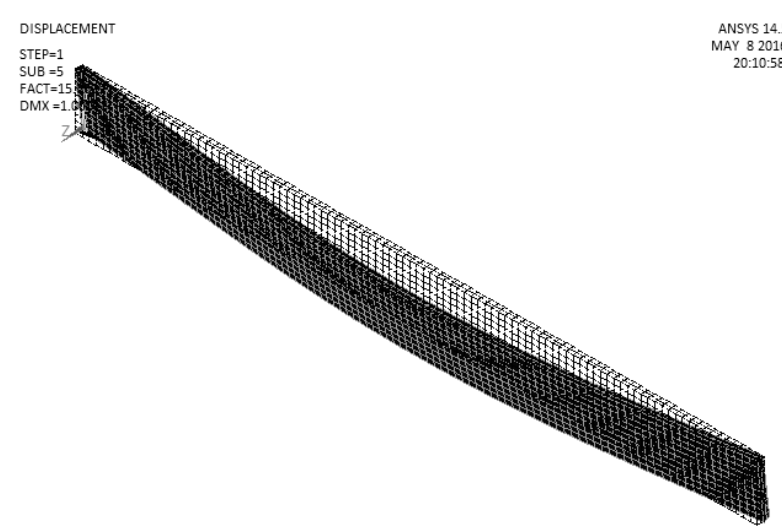

(a) Primeiro modo de flambagem $-F A C T=15,070$

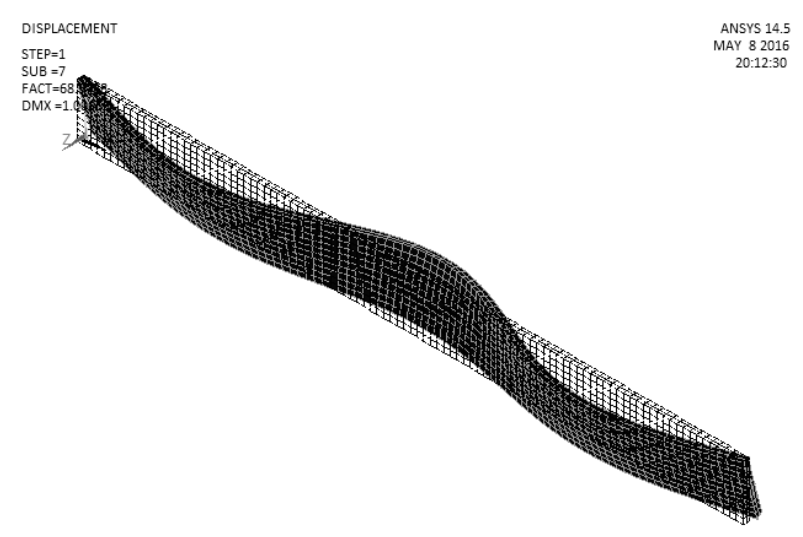

(c) Terceiro modo de flambagem $-F A C T=68,373$ pois o valor da carga crítica obtido foi o menor dentre os modelos. A suspensão com cabos retos mostrou-se a mais vantajosa, sobretudo quando ela é feita com balanços, de forma que não é introduzido na viga nenhum esforço de compressão associado à componente horizontal da força de tração nos cabos.

Lima (1995) apresentou os valores de carga crítica utilizando a formulação de RungeKutta. Para a situação de içamento com cabos retos sem balanços, a relação carga crítica/peso próprio (RCP) obtida por Lima (1995) foi de 2,82. Para o içamento com cabos inclinados sem balanços, RCP foi igual a 2,37. E, por fim, para cabos retos com balanços, RCP foi de 117,018. As diferenças obtidas podem ser atribuídas à natureza das análises realizadas e dos elementos adotados numericamente. Uma análise que permite verificar o comportamento volumétrico é mais precisa e representa melhor as reais condições de contorno às quais a viga está submetida.

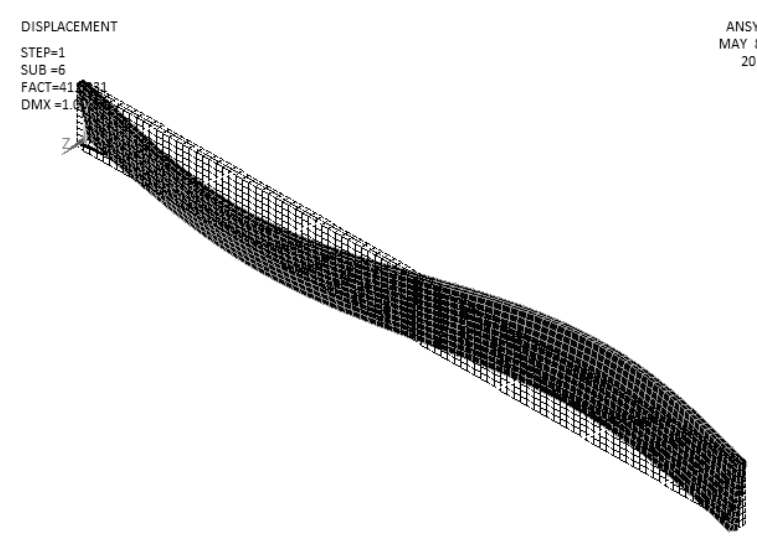

(b) Segundo modo de flambagem $-F A C T=41,033$

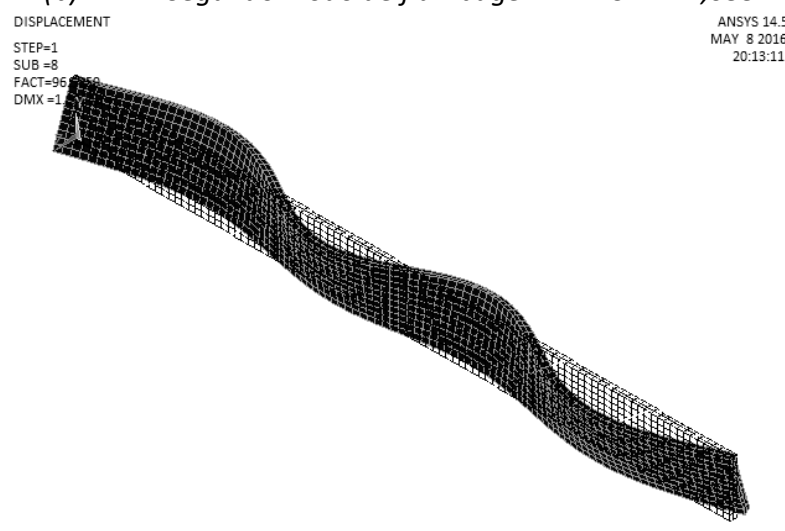

(d) Quarto modo de flambagem $-F A C T=96,536$

FIGURA 4: Modos de flambagem e suas respectivas cargas críticas.

FONTE: ANSYS (2017). 
TABELA 2: Carga crítica de instabilidade para cada caso analisado na fase transitória de suspensão e diferentes elementos

\begin{tabular}{lccc} 
CASO ANALISADO & \multicolumn{3}{c}{ FACT } \\
Fase transitória de suspensão & \multicolumn{2}{c}{ RCP - relação carga crítica/peso próprio } \\
\cline { 2 - 4 } & BEAM188 & SOLID65 & SOLID73 \\
\hline (a) Cabos retos sem balanços & 12,337 & 18,966 & 15,070 \\
\hline (b) Cabos inclinados sem balanços & 8,006 & 8,425 & 2,323 \\
\hline (c) Cabos retos com balanços & 106,940 & 152,070 & 127,970 \\
\hline & & \multicolumn{3}{c}{ FONTE: Autores (2017). }
\end{tabular}

\subsection{FASE DE PRÉ-SERVIÇO}

Além do içamento, a fase de pré-serviço considerou que a viga estava sobre apoios tipo garfo deformáveis e indeformáveis à torção com e sem balanços. As condições de contorno são de extrema importância em problemas de instabilidade. Por isso, elas são apresentadas em detalhes.

No primeiro caso analisado, considerando a viga platibanda sobre apoios tipo garfo indeformáveis à torção, para o modelo com elemento BEAM188, as restrições aos deslocamentos UY e UZ e ao giro ROTX foram aplicadas nas extremidades da viga. O deslocamento UX foi impedido apenas em uma extremidade. Para o modelo com elemento sólido SOLID65, manteve-se as mesmas restrições, com algumas diferenças. O deslocamento UZ foi impedido ao longo de toda a altura da seção transversal da viga nas duas extremidades; UX e UY foram impedidos apenas nos nós inferiores de uma extremidade e UY, nos nós correspondentes da outra extremidade. Valem as mesmas condições de contorno para o modelo com SOLID73, porém acrescenta-se a restrição ao giro ROTX nos nós de toda a altura da seção transversal da viga nas duas extremidades.

\section{A viga platibanda sobre apoios tipo garfo} deformáveis à torção foi a segunda análise. Neste modelo, a deformabilidade à torção foi considerada pela constante elástica à torção $\left(K_{\theta x}\right)$ que indica quão rígida é a ligação. $O$ valor de $K_{\theta x}$ é $2,9091 \times 10^{7}$ $\mathrm{kN} \cdot \mathrm{mm}$ e, no modelo numérico, as molas foram simuladas por elementos COMBIN40. O valor da constante elástica à torção foi igual ao adotado por Lima (1995).

$\mathrm{Na}$ análise com elemento BEAM188, a viga teve os deslocamentos UX, UY e UZ impedidos em uma extremidade e UY e UZ, na outra. As condições de contorno aplicadas aos modelos com elementos sólidos (SOLID65 e SOLID73) foram idênticas. Nos nós inferiores das duas extremidades, a translação UY foi impedida. No nó inferior intermediário de uma extremidade, restringiu-se UX e UZ. No mesmo nó da outra extremidade, restringiu-se UZ. Os nós externos à viga, aos quais a mola estava vinculada, tiveram o giro ROTX impedido tanto no modelo com BEAM188 quantos nos modelos com elementos SOLID65 e SOLID73.

O terceiro caso analisado considerou a viga platibanda com balanços sobre apoios tipo garfo indeformáveis à torção. O comprimento dos balanços era de 4,8 m. Para o modelo com elemento BEAM188, foram impedidos os deslocamentos UX, UY e UZ e o giro ROTX em um dos balanços e os deslocamentos UY e UZ no outro. Para a viga com elemento SOLID65, os deslocamentos UX, UY e UZ foram impedidos nos nós inferiores do primeiro balanço e UY e UZ nos nós correspondentes do segundo. As mesmas restrições aplicadas ao modelo com elemento SOLID65 valem para o modelo com elementos SOLID73 e acrescenta-se a restrição ao giro ROTX nos nós inferiores do primeiro balanço.

A Tabela 3 resume os valores de carga crítica obtidos para cada caso analisado na fase de pré-serviço de acordo com o tipo de elemento utilizado no modelo.

A Figura 5 apresenta as configurações deformadas dos quatro modos de flambagem para a viga platibanda sobre apoios tipo garfo indeformáveis à torção modelada com elemento BEAM188 e as respectivas cargas críticas de instabilidade lateral. 
TABELA 3: Carga crítica de instabilidade para cada caso analisado na fase de pré-serviço e diferentes elementos.

\begin{tabular}{|c|c|c|c|}
\hline \multirow{2}{*}{$\begin{array}{l}\text { CASO ANALISADO } \\
\text { Fase de pré-serviço }\end{array}$} & \multicolumn{3}{|c|}{$\begin{array}{c}\text { FACT } \\
\text { RCP - relação carga crítica/peso próprio }\end{array}$} \\
\hline & BEAM188 & SOLID65 & SOLID73 \\
\hline $\begin{array}{l}\text { (a) Viga platibanda sobre apoios tipo garfo } \\
\text { indeformáveis à torção }\end{array}$ & 20,658 & 24,657 & 23,955 \\
\hline $\begin{array}{l}\text { (b) Viga platibanda sobre apoios tipo garfo } \\
\text { deformáveis à torção }\end{array}$ & 17,870 & 15,123 & 13,802 \\
\hline $\begin{array}{l}\text { (c) Viga platibanda com balanços sobre apoios } \\
\text { tipo garfo indeformáveis à torção }\end{array}$ & 118,120 & 226,790 & 192,710 \\
\hline
\end{tabular}

FONTE: Autores (2017).

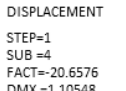$$
\text { (a) }
$$$$
\text { Primeiro modo de flambagem }-F A C T=20,658
$$

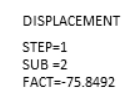

SUB $=2$
FACT $=75.8492$
DMX $=1.17922$

(c)

Terceiro modo de flambagem - FACT $=75,849$

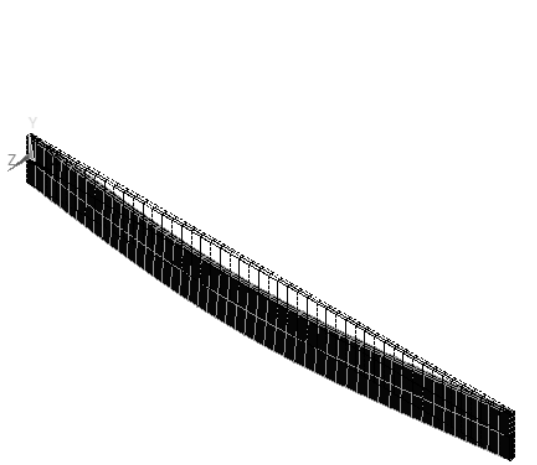

$\begin{array}{cl}\text { ANSYS 14.5 } & \text { DIPPLACEMENT } \\ \text { APR 25 2016 } & \text { STEP }=1 \\ \text { 20:42:10 } & \text { SUB } 3 \\ & \text { FACT=-48.1391 } \\ & \text { DMM }=1.21385\end{array}$

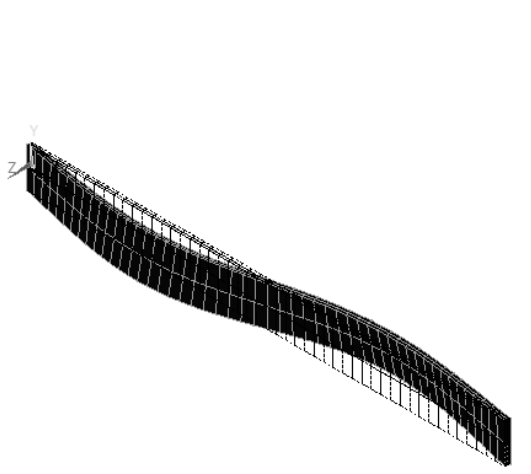

(b)

Segundo modo de flambagem $-F A C T=48,139$

\section{STEP $=1$
SUB $=1$ \\ SUB $=1$
FACT $=-103.8$
DMX $=1.27545$}

\section{ANSYS 14.5
APR 252016
$20: 40: 11$}

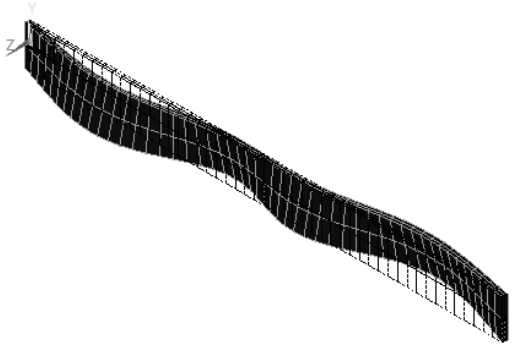

(d) Quarto modo de flambagem - FACT $=103,800$ FIGURA 5: Modos de flambagem e suas respectivas cargas críticas.

FONTE: ANSYS (2017).

Na fase de pré-serviço, os valores mais altos de carga crítica foram obtidos quando a viga encontrava sobre apoios indeformáveis e com balanços. A rigidez elevada desses apoios diminui ou quase elimina a possibilidade de giro da viga garantindo estabilidade à estrutura. Como esperado, a utilização de garfos desformáveis produziu uma carga crítica de instabilidade menor que a produzida pela utilização de garfos indeformáveis à torção.

Pela formulação de Runge-Kutta, Lima
(1995) obteve relação carga crítica/peso próprio (RCP) de 21,09 para a viga platibanda sobre apoios tipo garfo indeformáveis à torção e RCP de 18,19 para a viga platibanda sobre apoios tipo garfo deformáveis à torção. Os valores obtidos nas análises numéricas e apresentados na Tabela III mostraram uma boa aproximação com aqueles obtidos em Lima (1995).

Por fim, percebe-se que ocorreram diferenças entre as cargas críticas entre modelos com elemento de viga e com elementos sólidos. 
Essa diferença pode ser atribuída à natureza do elemento. BEAM188 é um elemento linear com comportamento diferente daqueles em três dimensões (SOLID65 e SOLID73).

\section{CONCLUSÕES}

O trabalho apresentou a análise de instabilidade na viga platibanda. Os valores de carga crítica obtidos na análise de instabilidade na viga platibanda foram coerentes com os valores correspondentes obtidos em formulações analíticas no trabalho de Lima (1995).

Dos casos de suspensão analisados, a maior relação entre carga crítica e peso próprio (RCP) foi obtida para a situação de cabos retos com balanços (RCP de 106,940 para o modelo com elemento BEAM188), ou seja, é o caso de içamento mais vantajoso e seguro para a viga. Em seguida, tem-se a suspensão com cabos retos sem balanços (RCP de 12,337 para o modelo com elemento BEAM188). O içamento mais crítico é aquele realizado com cabos inclinados sem balanços que apresentou o menor valor de carga crítica (RCP 8,006 para o modelo com elemento BEAM188).

$\mathrm{Na}$ fase de pré-serviço, a melhor condição de apoio para a viga é sobre apoios tipo garfo indeformáveis à torção na presença de balanços com RCP de 118,120 para o modelo com elemento BEAM188. Em seguida, tem-se a condição de viga sobre apoios tipo garfo indeformáveis à torção sem balanços (RCP de 20,658 para o modelo com elemento BEAM188). A condição menos vantajosa é a viga apoiada sobre garfos deformáveis à torção sem balanços que apresentou o menor valor de carga crítica (RCP igual a 17,870 para o modelo com elemento BEAM188).

Finalmente, dentre as situações analisadas, o içamento foi a mais crítica em termos de carga de instabilidade. As análises numéricas conduzidas neste artigo mostraram que a presença de balanços e a suspensão com cabos retos ou inclinados são parâmetros importantes para a segurança durante o manuseio da viga. Nesse sentido, sugere-se analisar sua influência e efeito na estabilidade da viga. Além deles, a presença de imperfeições geométricas iniciais e a excentricidade das alças de içamento podem ser ainda mais desfavoráveis à viga, justificando uma análise mais detalhada.

\section{AGRADECIMENTOS}

Agradecemos à CAPES pela concessão da bolsa de estudo no nível Mestrado Acadêmico a Maria Teresa Santos do Amaral Cardoso.

\section{REFERÊNCIAS BIBLIOGRÁFICAS}

ANSYS. v. 17.0. ANSYS, Inc.

BAIRAN, J. M.; CLADERA, A. Collapse of a precast concrete beam for a light roof. Importance of elastomeric bearing pads in the element's stability. Engineering Failure Analysis, v. 39, p. 188-199, apr., 2014.

GIRIJA, K.; MENON, D. Reduction in flexural strength in rectangular RC beams due to slenderness. Engineering Structures, v. 33, n. 8, p. 2398-2406, aug., 2011.

IMPER, R. R.; LAZLO, G. Handling and shipping of long span bridge beams. PCI Journal, v. 32, n. 6, p. 86-101, nov.-dec., 1987.

KRAHL, P. A. Instabilidade lateral de vigas pré-moldadas em situações transitórias. 2014. 208 f. Dissertação (Mestrado em Engenharia de Estruturas) - Escola de Engenharia de São Carlos, Universidade de São Paulo, São Carlos, 2014.

LIMA, M. C. V. Instabilidade lateral das vigas prémoldadas em regime de serviço e durante a fase transitória. 1995. 181 f. Dissertação (Mestrado em Engenharia de Estruturas) - Escola de Engenharia de São Carlos, Universidade de São Paulo, São Carlos, 1995.

LIMA, M. C. V. Contribuição ao estudo da instabilidade lateral de vigas pré-moldadas. 2002. $179 \mathrm{f}$. Tese (Doutorado em Engenharia de Estruturas) - Escola de Engenharia de São Carlos, Universidade de São Paulo, São Carlos, 2002.

MASSEY, C.; WALTER, K. R. The Lateral Stability of a Reinforced Concrete Beam Supporting a Concentrated Load. Building Science, v. 3, n. 3, p. 183-187, jan., 1969.

MAST, R. F. Lateral stability of long prestressed concrete beams - Part 1. PCI Journal, v. 34, n. 1, jan.-feb., 1989.

MAST, R. F. Lateral stability of long prestressed concrete beams - Part 2. PCI Journal, v. 38, n. 1, jan.-feb., 1993.

MOEN, C. D. Direct calculation of deflections and stresses in precast prestressed concrete beams during lifting. PCI Convention, Washington, DC, 2014. 
OESTERLE, R. G.; SHEEHAN, M. J.; LOTFI, H. R.; CORLEY, W. G.; ROLLER, J. J. Investigation of red mountain freeway bridge girder colapse. Arizona Department of Transportation, Bridge Group, Skokie, Illinois. CTL Group Project No. 262291, nov., 2007.

PLAUT, R. H.; MOEN, C. D. Analysis of Elastic, Doubly Symmetric, Horizontally Curved Beams during Lifting. Journal of Structural Engineering, v. 139, n. 1, p. 39-46, jan., 2013.

PLAUT, R. H.; MOEN, C. D. Stability of unbraced concrete beams on bearing pads including wind loading. Journal of Structural Engineering, v. 69, p. 246-254, jun., 2014.

STRATFORD, T. J.; BURGOYNE, C. J. Lateral stability of long precast concrete beams. Proceedings of the Institution of Civil Engineers - Structures and Bridges, v. 124, p. 169180, may, 1999.

SWANN, R. A.; GODDEN, W. G. The lateral buckling of concrete beams lifted by cables. The Structural Engineer, v. 44, p. $21-33,1966$.

TREMBLAY, R.; MITCHELL, D. Collapse during Construction of a Precast Girder Bridge. Journal of Performance of Constructed Facilities, v. 20, n. 2, p. 113125, may, 2006. 